\title{
A numerical investigation of snap-through in a shallow arch-like model
}

\author{
Yenny Chandra ${ }^{a}$, Ilinca Stanciulescu, ${ }^{\mathrm{a}, *}$, Lawrence N. Virgin ${ }^{\mathrm{b}}$, Thomas G. \\ Eason $^{\mathrm{c}}$, Stephen M. Spottswood ${ }^{\mathrm{c}}$ \\ ${ }^{a}$ Rice University, Department of Civil and Environmental Engineering, Houston, TX, \\ 7ro05, U.S.A \\ ${ }^{b}$ Duke University, Department of Mechanical Engineering, Durham, NC, 27708, U.S.A. \\ ${ }^{c}$ Air Force Research Laboratory, Structural Sciences Center, 2790 D. Street, WPAFB, \\ OH, U.S.A.
}

\begin{abstract}
Slender curved structures may experience a loss of stability called snapthrough, causing the curvature on part or all of the structure to invert inducing fatigue damage. This paper presents a framework for analyzing the transient responses of slender curved structures. A numerical study of snapthrough in a shallow arch-like model under periodic excitations is performed on a simplified model and on a detailed finite element model. The boundaries that separate the snap-through and no snap-through regions in the forcing parameters space are identified. Various post-snap responses are analyzed. The effects of initial conditions on the snap-through boundaries and post-snap responses are examined. Forcing parameters that lead to chaotic response are identified.
\end{abstract}

Keywords: snap-through, curved structures, transient loads, stability boundaries

\section{Introduction}

Advances in lightweight materials and modern performance requirements lead to increasingly lighter designs for the next generation aircraft, and, consequently, to more slender structural components. In such systems, the

*Corresponding author. Tel.: +1713 348 4704; fax: +1713 3485268.

Email address: ilinca@rice.edu (Ilinca Stanciulescu) 
slenderness of the structure greatly increases the risk of loss of stability. One type of loss of stability analyzed in this paper is snap-through where the structure jumps to a remote state equilibrium causing the curvature on part or all of the structure to invert (Figure 1a). At a snap-through event, the load-deflection diagram presents a jump, e.g., from point B to point C, as shown in Figure 1b.

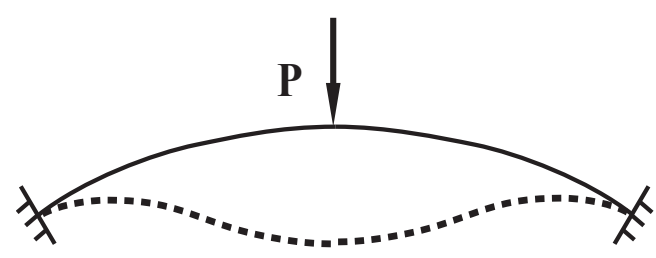

(a)

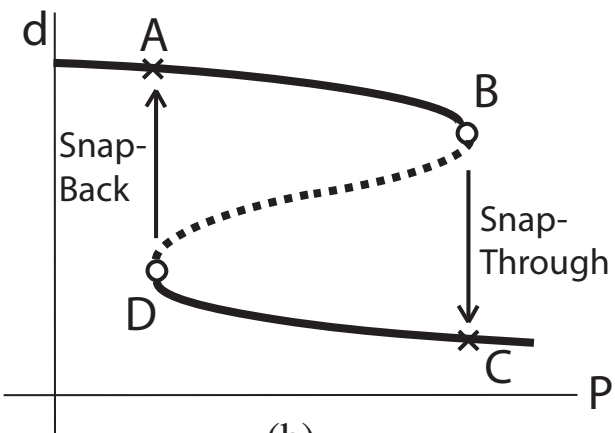

(b)

Figure 1: Snap-through buckling of shallow structures: (a) initial and post-snap configuration, (b) load-deflection curve.

In structures that have such load-deflection diagrams, multiple equilibrium configurations exist and dynamic excitations might cause oscillations between coexisting configurations and hence accelerate fatigue damage [1]. Examples of such structures are shallow curved panels (used in many basic structural components in an aircraft), pipelines, pressure vessels, arch bridges, submarines, and many more. Therefore the characterization of their dynamic behavior is very important for a more reliable design of these structures.

In this paper, we present a framework for analyzing the dynamic responses of slender curved structures. A numerical study of snap-through in a shallow arch-like model under periodic excitations is performed on a simplified model and on a detailed finite element model. Relevant features useful in characterizing the transient behavior of shallow arches are identified. In previous work, a discrete system that experiences snap-through buckling under forced vibrations was analyzed [2]. The authors presented experimental results and numerical simulations of a single degree of freedom (SDOF) system under periodic excitation. In order to gain further insights on systems that experience snap-through buckling, we extend the study to continuous systems, 
e.g., shallow arches.

The dynamic response of curved structures has been studied by many authors. Mettler [3, 4] presented the analysis of stability and vibration of a sinusoidal arch under periodic loading. Hsu [5], Humphreys [6], and Lock [7] studied the behavior of shallow arches subjected to step and impulsive loading. Huang [8] investigated the behavior of elastic shallow structures under high-frequency periodic loading. Poon [9] presented the study of snapthrough on a clamped-clamped buckled beam under harmonic excitation.

The structures examined in this paper are shallow arches with pin supports and a very specific geometry (sinusoidal shape) and loading (distributed, sinusoidal). This configuration and loading combination allows us to simplify the analysis to a one degree of freedom system [10]. Numerical simulations are performed for the simplified model. Simulations with a full finite element model identify the parameter ranges for which the simplified analysis is valid. We also discuss pertinent quantities, such as, number of snap-through events per forcing cycle and kinetic energy, useful in illuminating some of the transient behavior of shallow arches. In addition, the region of chaotic responses is also identified. No comparison with experimental data will be performed due to the difficulties in setting up the experiments for this geometry and loading pattern. The numerical simulations are performed with Matlab and the Finite Element Analysis Program (FEAP), a research code that includes most commonly used finite elements and solvers and provides a reliable framework for developing and implementing new user formulations [11].

This paper is organized as follows. In Section 3, we present the static analysis of the shallow arches. The equilibrium paths (primary and bifurcated) are identified and the stability of the solutions is discussed. Section 4 analyzes the transient responses of the structures under periodic excitations and identifies the important features of the transient behavior. A summary of our findings is presented in the concluding section.

\section{Governing equations}

The continuum model that we analyze is an arch with pinned supports, sinusoidal initial shape and subjected to a sinusodial static load distribution (Figure 2). The arch is homogeneous with Young's modulus $E$, uniform cross-section $A$, moment of inertia $I$, damping $c$, and mass per unit length $\mu$. For this specific geometry and loading (sinusoidal), a simple model for the 
system can be derived in terms of a single degree of freedom (the coordinate of the midpoint).

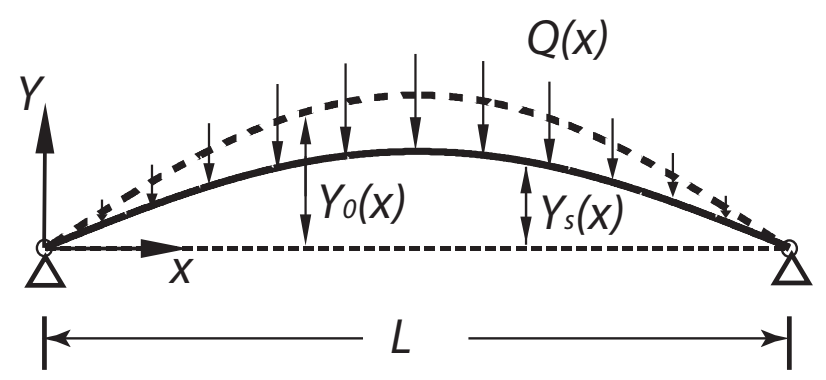

Figure 2: Geometry of a shallow arch with sinusoidal shape and sinusoidal loading.

In the simplified analysis briefly introduced here, the axial and rotary inertia terms are neglected. For full details the interested reader is referred to [10]. Denoting by $Y_{0}(X)$ the initial shape, $Q(X)$ the distributed load, $Y_{s}(X)$ the equilibrium configuration, and $Y(X, T)$ the configuration of the arch at time $T$, the equation of motion can be written as:

$$
\mu \frac{\partial^{2} Y}{\partial T^{2}}+c \frac{\partial Y}{\partial T}+E I \frac{\partial^{4}\left(Y-Y_{0}\right)}{\partial X^{4}}+M \frac{\partial^{2} Y}{\partial X^{2}}=-Q
$$

where

$$
M=\frac{E A}{2 L} \int_{0}^{L}\left[\left(\frac{\partial Y_{0}}{\partial X}\right)^{2}-\left(\frac{\partial Y}{\partial X}\right)^{2}\right]
$$

$M$ expresses the axial force due to mid-axis stretching or compression. The boundary conditions are

$$
Y=0, \quad E I \frac{\partial^{2}\left(Y-Y_{0}\right)}{\partial X^{2}}=0, \quad \text { at } X=0, L
$$

Let us define the dimensionless variables as

$$
\begin{gathered}
x=\frac{X}{L}, \quad y=\frac{Y}{2 r}, \quad y_{0}=\frac{Y_{0}}{2 r}, \quad y_{s}=\frac{Y_{s}}{2 r}, \\
\beta=\frac{c L^{2}}{\pi^{2} \sqrt{E I \mu}}, \quad t=\pi^{2} T \sqrt{\frac{E I}{\mu L^{4}}}, \quad q=\frac{Q L^{4}}{2 \pi^{4} E I r},
\end{gathered}
$$


where $r=\sqrt{\frac{I}{A}}$. Then Eq. (2) becomes

$$
\pi^{4} \frac{\partial^{2} y}{\partial t^{2}}+\pi^{4} \beta \frac{\partial y}{\partial t}+\frac{\partial^{4}\left(y-y_{0}\right)}{\partial x^{4}}+m \frac{\partial^{2} y}{\partial x^{2}}=-\pi^{4} q
$$

where

$$
m=2 \int_{0}^{1}\left[\left(\frac{\partial y_{0}}{\partial x}\right)^{2}-\left(\frac{\partial y}{\partial x}\right)^{2}\right] d x
$$

The dimensionless equilibrium configuration $y_{s}(x)$ satisfies

$$
\frac{\partial^{4} y_{s}}{\partial x^{4}}-\frac{\partial^{4} y_{0}}{\partial x^{4}}+\gamma^{2} \frac{\partial^{2} y_{s}}{\partial x^{2}}=-\pi^{4} q
$$

where

$$
\gamma^{2}=2 \int_{0}^{1}\left[\left(\frac{\partial y_{0}}{\partial x}\right)^{2}-\left(\frac{\partial y_{s}}{\partial x}\right)^{2}\right] d x
$$

Then, the initial sinusoidal shape and the sinusoidal loading are represented as

$$
y_{0}(x)=\lambda \sin \pi x, \quad q(x)=p \sin \pi x, \quad \lambda>0, p>0
$$

Assuming rectangular cross-section, $Y_{0}(X=L / 2)=0.57735 \lambda h$, where $h$ is the thickness of the beam. The configuration of the beam is assumed to be a sinusoidal shape

$$
y(x, t)=d(t) \sin \pi x
$$

where $d$ is the coordinate of the midpoint measured from the origin. With these notations, a simplified dimensionless one degree of freedom model that can represent only symmetric deformed configurations is obtained:

$$
\ddot{d}+\beta \dot{d}+d^{3}+\left(1-\lambda^{2}\right) d+p-\lambda=0 .
$$

For a shallow arch, we assume that $\left(\frac{\partial Y}{\partial X}\right)^{2} \ll 1$ and $\left(\frac{\partial Y_{0}}{\partial X}\right)^{2} \ll 1$, which implies $\left(\frac{d r}{L}\right)^{2} \ll 1$ and $\left(\frac{\lambda r}{L}\right)^{2} \ll 1[12]$. 


\section{Static Analysis}

A typical load-deflection diagram of a shallow arch is shown in Figure 1(b). For certain levels of steady loading, multiple stable equilibria may exist and the primary solution path has intervals corresponding to unstable equilibrium configurations. These equilibria, both stable and unstable, play an important role in the dynamic behavior. In this section, we determine the equilibrium paths of the arches under consideration $\left(y_{s}<2 h\right)$. For certain geometries, branching of the primary solution path occurs. These secondary paths (corresponding to asymmetric equilibrium configurations) are also obtained. The dimensions of the arch used in this paper are listed in Table 1 and the material properties are listed in Table 2. For the choice of geometry taken here, $\frac{r}{L}=1.6 \times 10^{-3}$, which ensures that $\left(\frac{d r}{L}\right)^{2} \ll 1$ and $\left(\frac{\lambda r}{L}\right)^{2} \ll 1$.

Table 1: Dimensions

\begin{tabular}{|l|r|}
\hline Dimensions & Values \\
\hline Length $L[\mathrm{~mm}]$ & 304 \\
Width $(d)[\mathrm{mm}]$ & 25.4 \\
Thickness $(t)[\mathrm{mm}]$ & 1.75 \\
\hline
\end{tabular}

Table 2: Material properties

\begin{tabular}{|l|r|}
\hline Properties & Values \\
\hline Young's Modulus $\left[\mathrm{N} \mathrm{mm}^{-2}\right]$ & 2300 \\
Poisson's Ratio & 0.37 \\
Density $\left[\mathrm{g} \mathrm{mm}^{-3}\right]$ & $1.21 \times 10^{-3}$ \\
\hline
\end{tabular}

Note that the system represented in Figure 2 also has asymmetric equilibrium configurations for certain values of the parameter $\lambda$. Since the simplified model cannot capture this behavior, we also analyze the system using the finite element method (FEM). Comparing the results obtained with the two approaches will then identify the parameter ranges for which a simplified analysis can be used without neglecting important features of the physical system.

Figures 3 to 6 show the load deflection diagram along with the stability of the solutions for increasing values of arch heights. An arch with a very shallow initial configuration buckles to a symmetric remote equilibrium at the limit point (i.e., a point of local maximum on the load-deflection path) (Figure 3(a)). The stability of the solution may change along the equilibrium path. The dashed line in Figure 3(a) represents the unstable part of the solution path. The changes in stability can be shown by the change in sign of the lowest eigenvalues of the stiffness matrix. The eigenvalues plot for the two lowest eigenvalues (obtained using FEM) shows that after the limit point the solution becomes unstable (one negative eigenvalue) and it restabilizes after the next limit point (Figure 3(b)). 


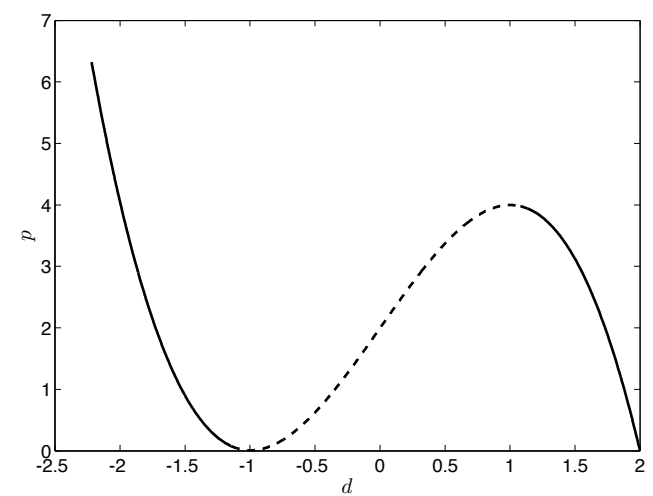

(a)

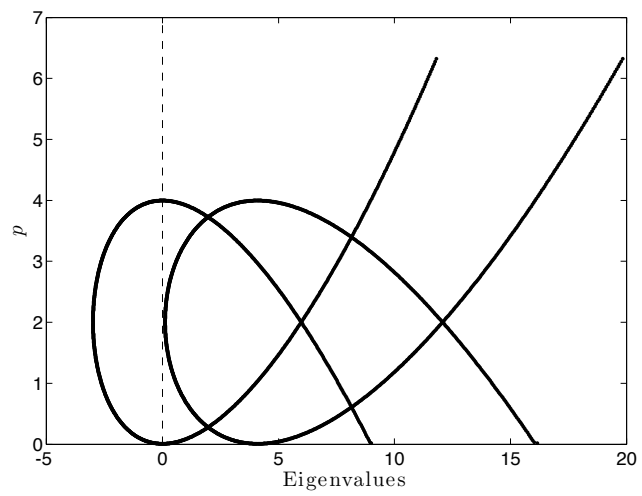

(b)

Figure 3: Solution path for $\lambda=2$ : (a) equilibrium path and (b) the first two eigenvalues.

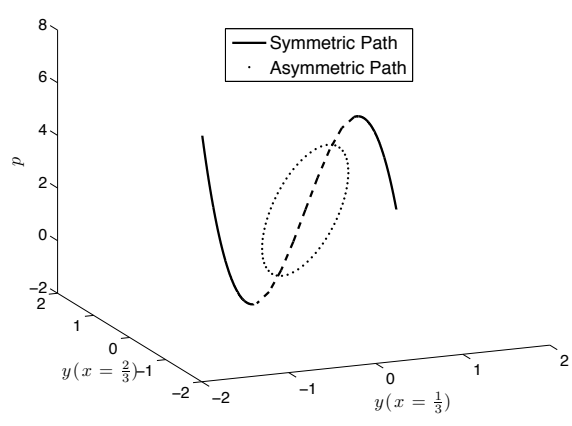

(a)

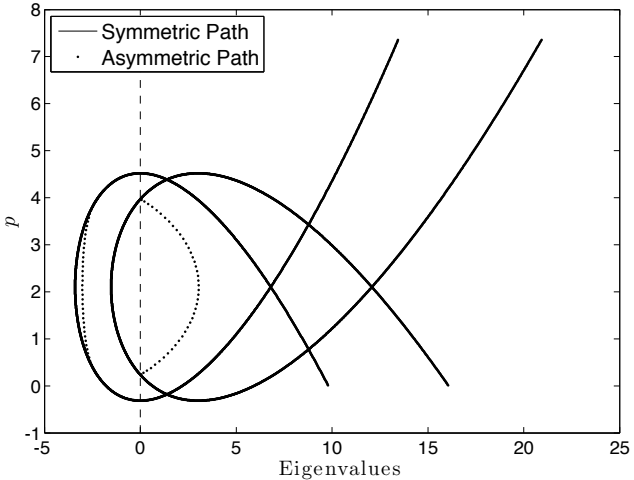

(b)

Figure 4: Solution path for $\lambda=2.1$ : (a) equilibrium path and (b) the first two eigenvalues.

A slightly deeper arch $(\lambda=2.1)$ has asymmetric configurations with the bifurcation point (i.e., the intersection of two or more equilibrium paths) appearing after the limit point (Figure 4(a)). The horizontal axes in Figure 4 (a) are the $y$ coordinates at $x=1 / 3$ and $x=2 / 3$. The eigenvalues plot (Figure 4(b)) shows that at configurations on the symmetric path, the lowest eigenvalue is negative after the critical load is reached and the symmetric solution becomes unstable. Then the second lowest eigenvalue becomes negative when the branching into asymmetric solutions appears. From the two lowest eigenvalues at equilibrium states on the asymmetric path, one is always positive, and the other is always negative along the path. At load 


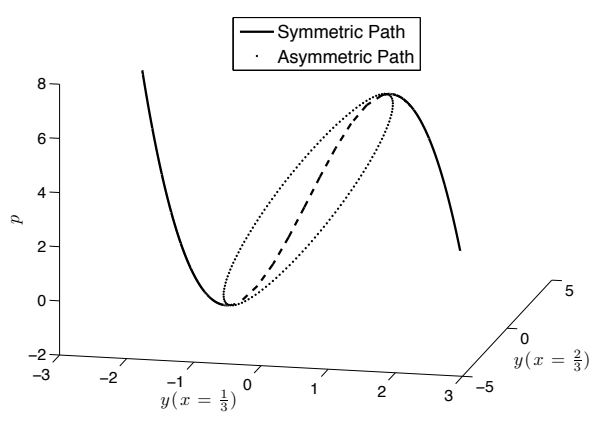

(a)

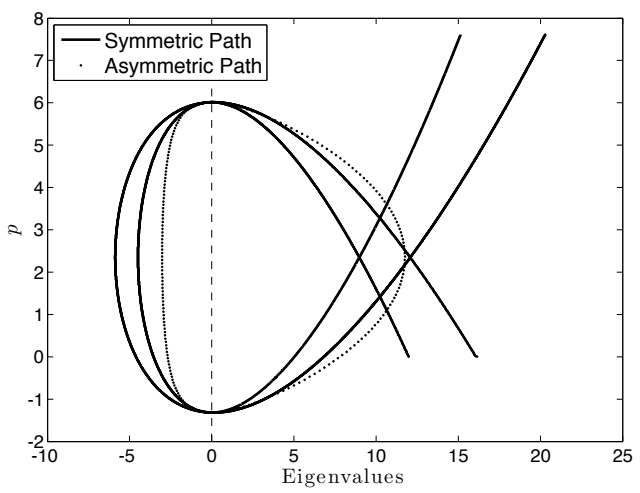

(b)

Figure 5: Solution path for $\lambda=2.3452$ (coincident configuration): (a) equilibrium path and (b) the first two eigenvalues.

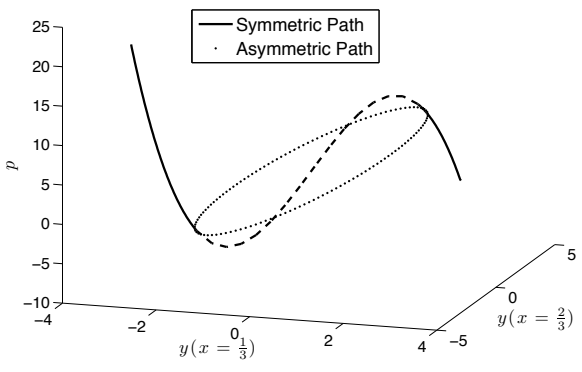

(a)

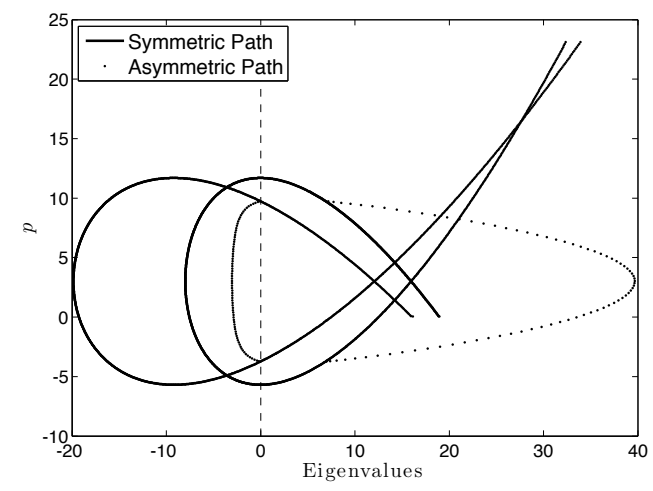

(b)

Figure 6: Solution path for $\lambda=3$ : (a) equilibrium path and (b) the first two eigenvalues.

levels where asymmetric configurations exist, a total number of five solutions coexist (two are stable and three are unstable).

As $\lambda$ is increased further, it will eventually reach a value that corresponds to a geometry for which the bifurcation point coincides with the limit point $(\lambda \approx 2.3452, Y(X=L / 2) \approx 1.35 h)[10]$ (Figure $5(\mathrm{a}))$. In what follows we will refer to this as the coincident configuration. The two lowest eigenvalues of the symmetric path become negative at the same time (Figure 5(b)). For this beam geometry, the eigenvalues also become positive at the same time. Again, of the two lowest eigenvalues for the asymmetric path, one is always 
positive, and the other is always negative along the path.

For an arch taller than the coincident configuration $(\lambda=3)$, the bifurcation occurs before the limit point and the structure will jump to a remote configuration soon after the bifurcation point (Figure 6(a)). The two lowest eigenvalues that correspond to the equilibrium configurations on all solution branches are shown in Figure 6(b). For configurations on the primary path, the lowest eigenvalue becomes negative when the asymmetric solutions appear, then the second eigenvalue becomes negative when the limit point is reached. Therefore, after the solution branches and two asymmetric solution paths appear, the symmetric solution becomes unstable and remains so until the asymmetric branches reconnect to the primary path (portion represented by a dashed line in Figure 6(a)). Maurini [13] presented similar discussion on post-buckling behavior of a simply supported straight beam with end-shortening. Their analysis used a nonlinear elastica approach and was restricted to the study of equilibrium configurations.

\section{Dynamic Analysis}

This investigation focuses on characterizing the dynamic behaviour of the arches and its dependence on the forcing parameters. Of relevance and providing significant insight into the transient behavior is the identification of the boundaries that separate the snap-through and no snap-through regions, i.e., the snap-through boundary. Various post-snap responses and the frequency of snap-through events (strongly related to fatigue) are examined. We also comparatively discuss the results obtained using the single degree of freedom model and the FE model. Further, we identify the region of chaotic responses in the forcing parameters space.

The equation of motion of the one-degree-of-freedom model of the structure can be seen in Eq. 11. If a dynamic load that excites only mode 1 is added, the equation of motion becomes

$$
\ddot{d}+\beta \dot{d}+d^{3}+\left(1-\lambda^{2}\right) d+p-\lambda=P(t)
$$

where $P(t)$ is the time dependent loading. A fourth-order Runge-Kutta (RK4) time stepping scheme is used to integrate Eq. (12) and obtain the forced dynamic response of the system. We also compare the results obtained using the one-dimensional model and FEM. For the FEM results presented in this paper, we use a beam formulation based on the Euler Bernoulli the- 
ory but extended to large deformations. From the mesh refinement study performed, 100 elements are used in the simulations.

\subsection{Influence of arch rise}

First, we examine the influence of the arch rise $(\lambda)$ on the snap-through boundary for arch configurations that snap-through at the limit point $(\lambda \leq 2.3452)$. We consider the system of Equation (12) with $\beta=0.2$ and subjected to harmonic loading: $P(t)=F \sin (\omega t)$, with the equilibrium configuration as the initial condition. In this case, the forcing parameters of interest are the amplitude $F$ and the frequency $\omega$. In this parameter space we sweep through $F$ and $\omega$ values, and solve the system for each such pair using the RK4 method. The arch is considered to be undergoing snap-through if $d \leq 0$ at any time during the simulations. This criterion is chosen due to the symmetry with respect to the horizontal line. If an arch experiences snapthrough, the snap-through responses are grouped into three categories: (1) reverted snap-through, (2) inverted snap-through, and (3) persistent snapthrough. Reverted snap-through includes cases when the arch experiences several snap-through events and in the end settles and oscillates around the original configuration. When the arch snaps-through (one or more times) but later oscillates around the remote configuration, we categorize the case as inverted snap-through. We call persistent snap-through the case in which the arch experiences snap-through and snap-back continuously. Persistent snap-through behavior clearly exacerbate fatigue faster than the other types.

Snap-through boundaries for two different arch configurations are shown: (1) $\lambda=2$ (Figure $7(\mathrm{a})$ ) and (2) $\lambda=2.3542$ (Figure $7(\mathrm{~b})$ ). For $\lambda=2$, we use $p=2$ while for $\lambda=2.3452, p=2.3452$ is used in Equation (12). These values are chosen for convenience in the computation of the initial equilibrium configuration. The two arch configurations have similar snap-through boundary profiles (Figure 7 ). For $\lambda=2$, the dimensionless linear natural frequency is 2.45 and for $\lambda=2.3452$, it is 3.0. Since these systems exhibit softening spring behavior, it is expected that the smallest $F$ for the arch to snap-through occurs at forcing frequencies slightly below these natural frequencies [1]. Moreover, for both configurations, the persistent snap-through occurs much more frequently than either the inverted or the reverted snapthrough. The reverted and the inverted snap-through are observed only in the region close to the snap-through boundaries and predominantly in the region of large forcing frequencies. The snap-through boundaries for all cases 


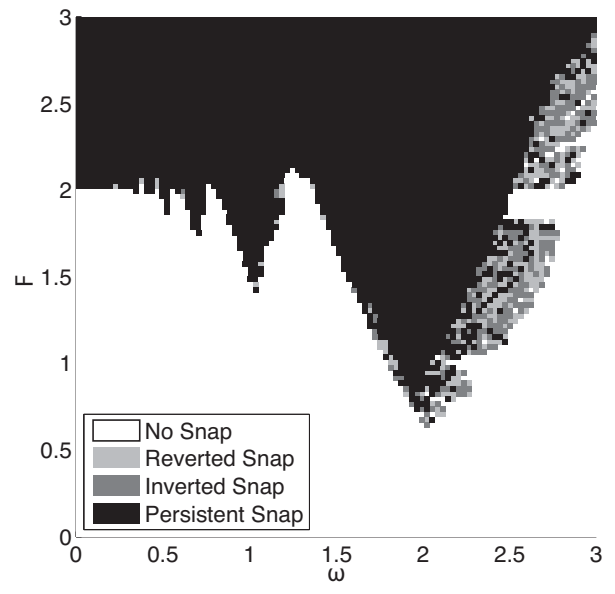

(a)

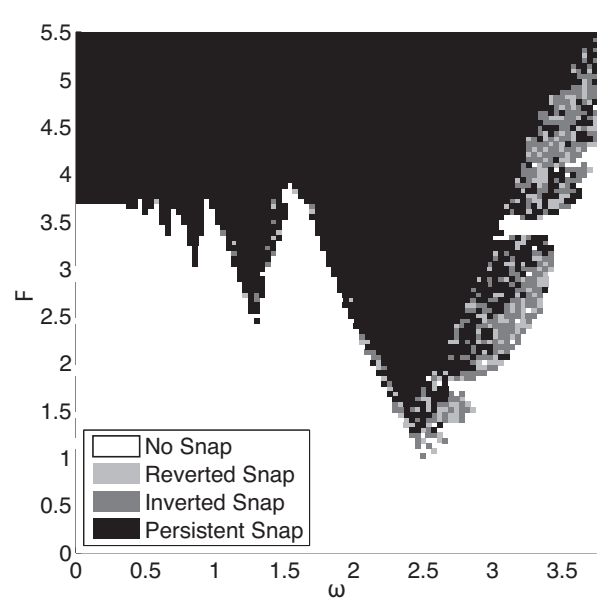

(b)

Figure 7: Snap-through boundaries and classification of the responses for systems with $\lambda \leq 2.3452$ : (a) $\lambda=2$, (b) $\lambda=2.3452$ (coincident configuration). Similar profiles are observed.

where the arch jumps to the remote configuration at the limit point are qualitatively similar.

\subsection{Number of forcing cycles needed to snap-through}

In addition to information about the type of snap-through that occurs, information on how soon (i.e., after how many forcing cycles) the arch undergoes snap-through under certain periodic excitation is also important for fatigue analysis. For most of the forcing parameters considered, when snapthrough occurs, the structure experiences the first snap-through in less than four forcing cycles (Figure 8). Only for parameters in the region close to the snap-through boundary, snap-through occurs after more than sixteen forcing cycles.

\subsection{Comparison with detailed FE model}

The simplified model has significant advantages in terms of speed and memory needed during simulations. However, since it only has one-degreeof-freedom, it is only able to capture the first symmetric mode and it is unable to correctly identify the snap-through load due to the asymmetric mode, i.e., it is unable to accurately capture the snap-through boundary for $\lambda>2.3542$. Recall that for $\lambda>2.3542$, the bifurcation point occurs before the 


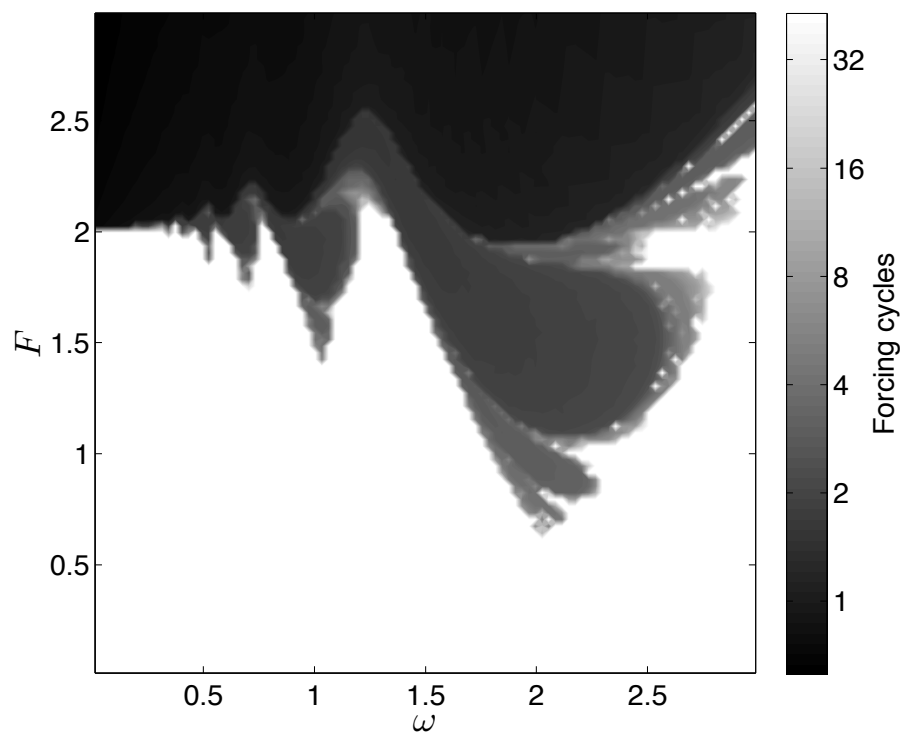

Figure 8: The number of forcing cycles needed for the arch to snap-through $(\lambda=2)$. Most cases show the structure snaps-through in less than four forcing cycles.

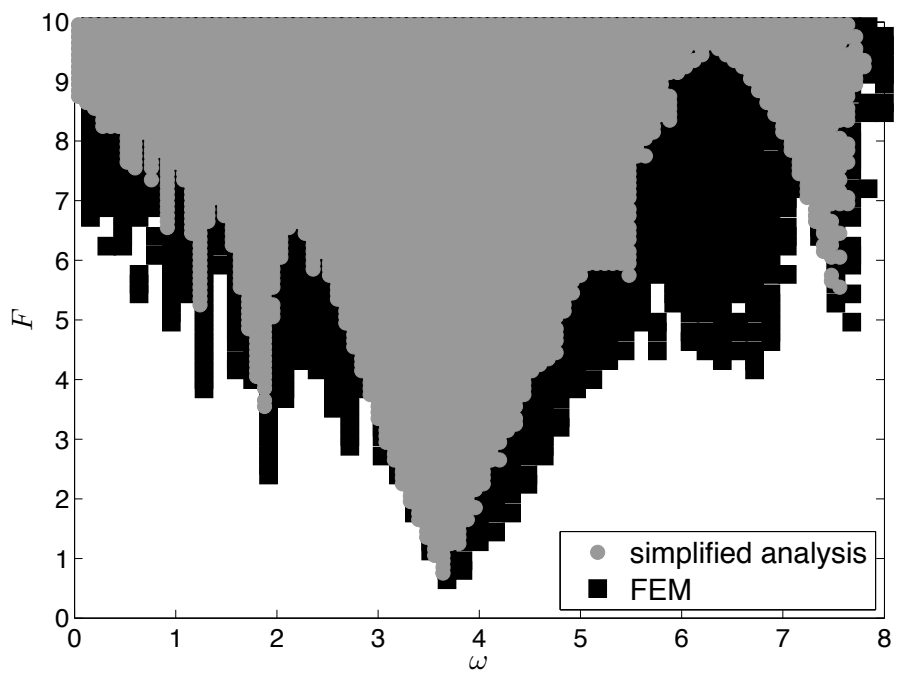

Figure 9: Snap-through boundary determined with the simplified analysis (gray) and full FE model (black). The FE model shows more forcing parameters cause the arch to snapthrough in comparison with the one degree of freedom model $(\lambda=3, p=3, \beta=0)$. 
limit point and the arch should undergo snap-through close to the bifurcation point (symmetric equilibrium configurations after bifurcation are unstable). Figure 9 shows how the identified snap-through boundaries differ when the simplified model (gray) and a full FE model (black) are used $(\lambda=3, p=3)$. In this study the damping term is ignored to eliminate the difference that results from translating the scalar value of damping used in the one-degreeof-freedom model to a damping matrix in multiple degree-of-freedom used in the FE model.

The simulations performed using the FE model identify additional forcing parameters ranges that cause the arch to snap-through beyond those identified with the simplified model. Therefore for cases where higher modes needed to be taken into account (i.e., when the bifurcation occurs prior to the limit point), it is necessary to use tools that are capable of considering more modes (e.g., FEM). For systems for which experimental data can also be available, additional modes beyond the capability of the simplified model are always necessary due to the presence of asymmetries in experiments.

\subsection{Effects of initial conditions}

Next, we investigate the effect of initial conditions on the snap-through boundaries (Figure 10(a)). The region filled with black color in Figure 10(a) corresponds to the forcing parameters that lead to snap-through when the system is excited from the equilibrium configuration; the gray color shows additional $(F, \omega)$ pairs for which the arch snaps-through when it is excited from some other random initial conditions. Additional initial conditions will likely expand the snap-through region more. Therefore, it is important to consider the variability of the initial conditions in identifying the safe region where snap-through does not occur.

The initial conditions influence not only whether the arch snaps-through, but also the type of snap-through. For a pair $(F, \omega)$, the arch might have different types of snapped-through responses for different initial conditions (Figure 10(b)). Therefore, identifying the different snap-through cases that can occur for each specific pair $(F, \omega)$ is important in order to determine the scenarios that lead to a higher fatigue risk. We solve Equation (12) using multiple initial conditions. Based on the types of snap-through obtained with the set of initial conditions we used, the forcing parameters space is divided into seven regions: (1) no snap-through, (2) no snap-through or inverted/reverted snap-through, (3) inverted/reverted snap-through, (4) no snap-through or 


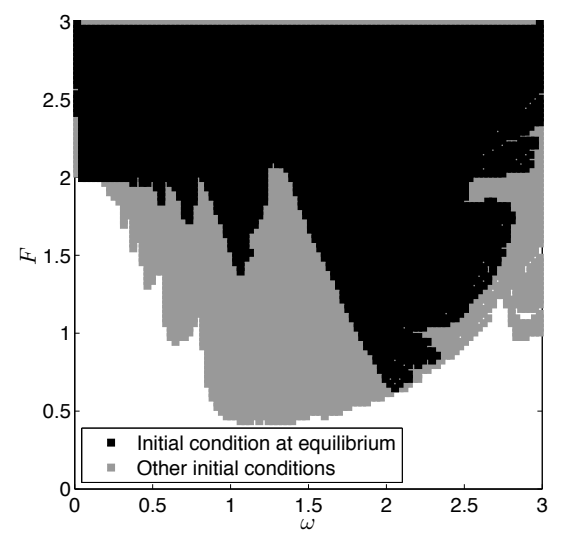

(a)

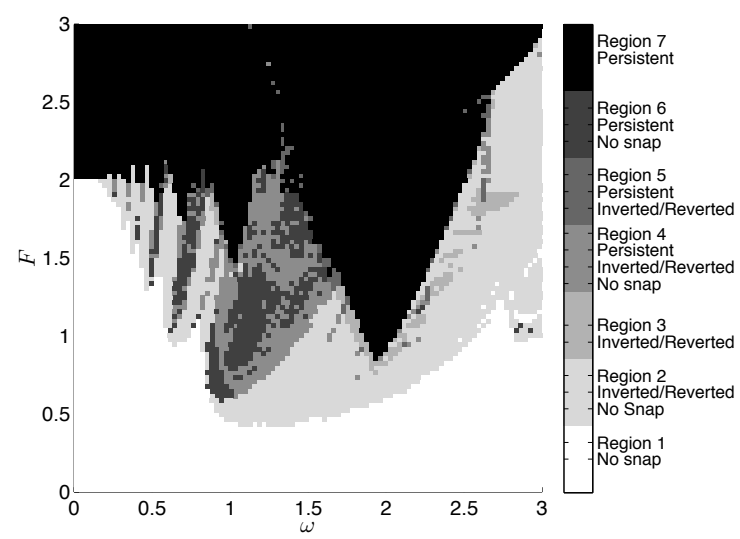

(b)

Figure 10: Effects of initial conditions on: (a) snap-though boundary, (b) the type of snapthrough. Snap-through boundaries expand when more initial conditions are considered. Different initial conditions may result in different types of snap-through for the same forcing parameters.

persistent snap-through, (5) no snap-through, inverted/reverted, or persistent snap-through, (6) inverted/reverted or persistent snap-through, and (7) persistent snap-through only. Region (1) is the region with the lowest fatigue risk, regions (2) and (3) can be considered as low fatigue risk regions, (4), (5), and (6) are regions with medium risk, and region (7) is a high risk region for the structure since all initial conditions tested lead to persistent snap-through.

In order to further discuss the influence of initial conditions on snapthrough responses, we choose two pairs of forcing parameters as examples: (1) $F=1.2, \omega=0.105$ (Figure $11(\mathrm{a})$ ), and $(2) F=1.2, \omega=1.515$ (Figure $11(\mathrm{~b}))$. We solve the system for each pair of initial position and initial velocity values using the RK4 method. For $F=1.2, \omega=0.105$, two possible snap-through types are observed: reverted and inverted snap-through. The regions for different snap-through types are easily identified in the space of initial conditions. Note that regions of reverted and inverted snap-through alternate and are well-defined. For some other forcing parameters, the different regions of snap-through type in the initial conditions parameter space might not be as easily identified, e.g., for $F=1.2, \omega=1.515$ (Figure 11(b)). For this pair of forcing parameters, three possible responses (inverted, reverted, and persistent snap-through) are identified but the different regions 
are not clearly separated.

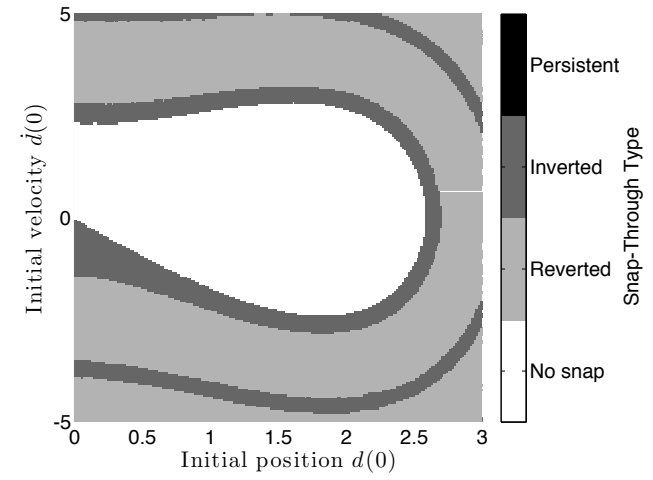

(a)

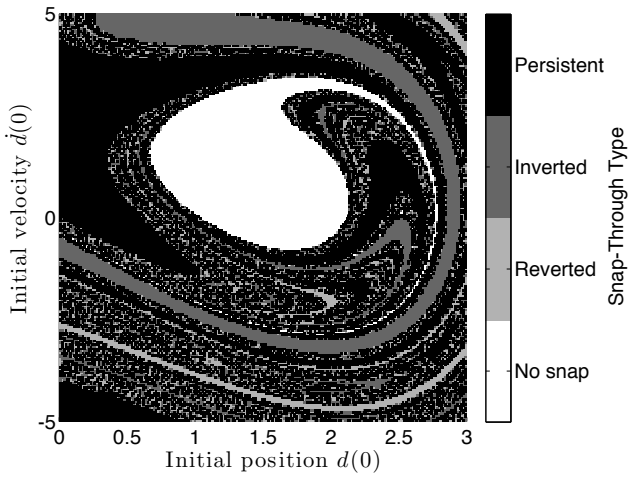

(b)

Figure 11: Snap-through boundaries in initial conditions parameters space for $\lambda=2$ : (a) $F=1.2 \omega=0.105$, (b) $F=1.2, \omega=1.515$.

Further study shows that even if the arch experiences the same type of snap-through (inverted, reverted, or persistent) regardless of the initial conditions, the post-snap responses might be very different depending on the forcing parameters. Consequently, the effect on fatigue will vary. Figures 12 to 14 show a collection of the responses of the arch $(\lambda=2)$ for some forcing parameters after the transients are given sufficient time to decay. In Figure 12 , the equilibrium paths are superimposed with the phase trajectories to illustrate whether the arch oscillates solely about one of the configurations or whether it snaps-through continuously between the two configurations. We can identify that Figures 12(a) to 12(f) are inverted snap-through cases, while Figures $12(\mathrm{~g})$ to $12(\mathrm{l})$ are the persistent snap-through cases. Since the arch has pinned supports and the stable equilibria are symmetric with respect to the horizontal line $(y(x)=0$ for all $x)$, for every inverted snap-through case, there is a corresponding reverted snap-through scenario.

The time series details and the phase plots of the responses that correspond to Figure 12 are shown in Figures 13 and 14, respectively. The persistent snap-through responses are sometimes symmetric about the horizontal line (Figures 13(g), 13(h), 13(i) and13(l)) but sometimes are biased toward one of the stable configurations (Figures 13(j), 13(k)). The use of Poincaré sections is a useful tool in nonlinear dynamics. Poincaré sections are obtained by stroboscopically sampling a trajectory at intervals of the 


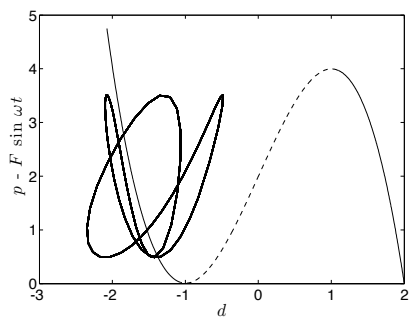

(a)

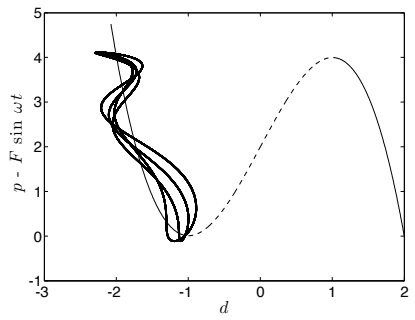

(d)

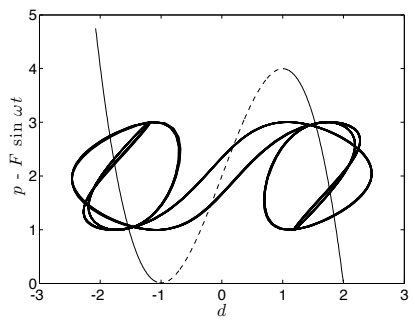

(g)

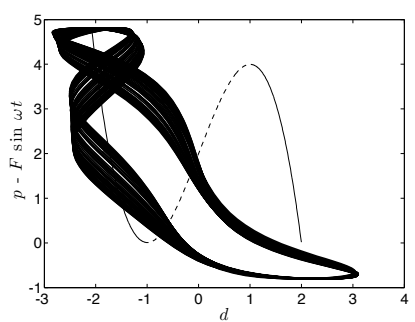

(j)

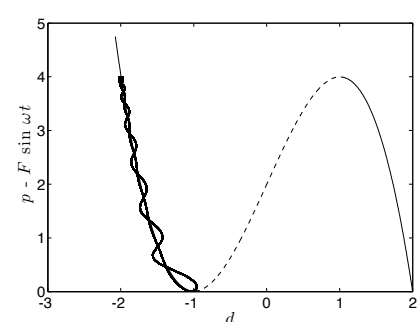

(b)

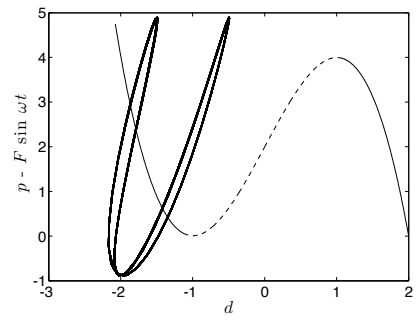

(e)

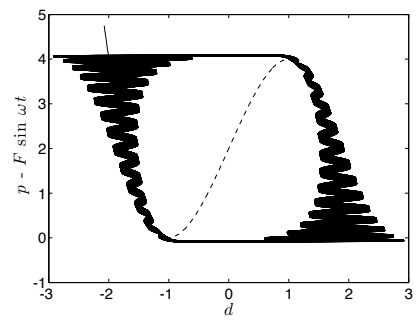

(h)

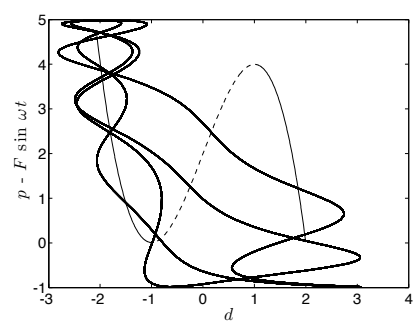

(k)

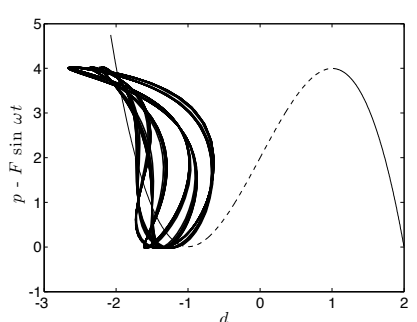

(c)

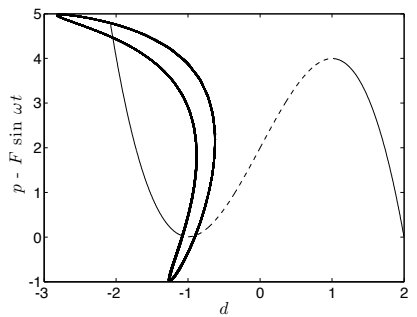

(f)

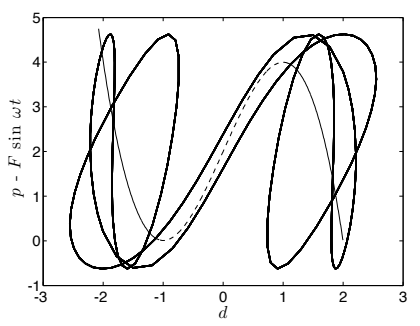

(i)

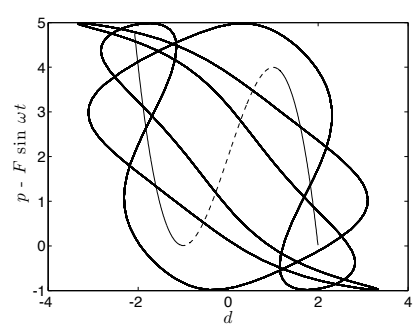

(1)

Figure 12: Time series superimposed on the restoring force $(\lambda=2$; sinusoidal load): (a) $F=1.515, \omega=2.955,(\mathrm{~b}) F=1.995, \omega=0.165$, (c) $F=2.025, \omega=1.155$, (d) $F=2.115, \omega=0.585$, (e) $F=2.895, \omega=2.985$, (f) $F=2.985, \omega=1.125$, (g) $F=1.005$, $\omega=1.905$, (h) $F=2.085, \omega=0.075$, (i) $F=2.655, \omega=2.595$, (j) $F=2.805, \omega=0.795$, (k) $F=2.985, \omega=0.435$, (l) $F=2.985, \omega=1.185$. 


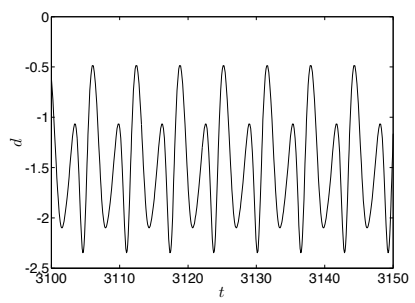

(a)

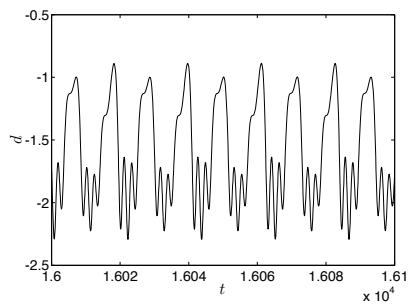

(d)

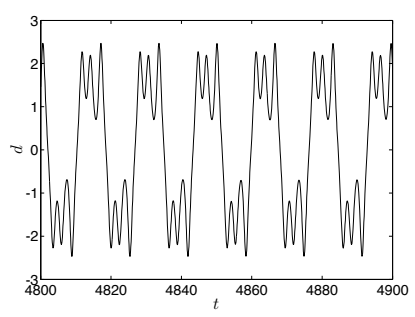

(g)

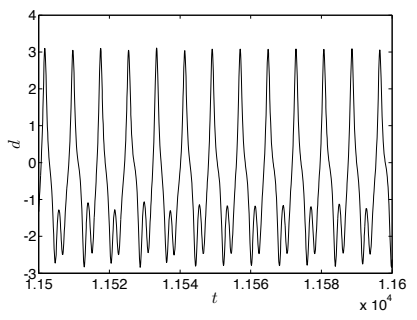

(j)

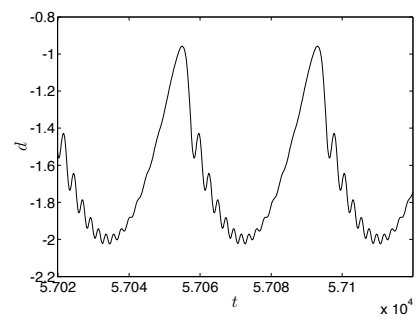

(b)

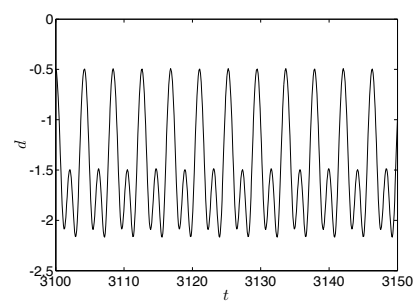

(e)

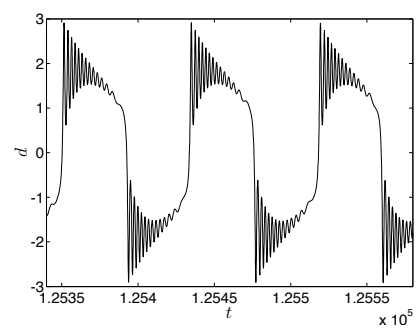

(h)

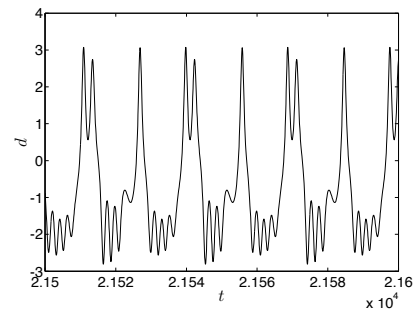

(k)

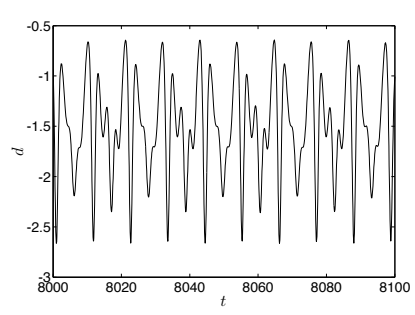

(c)

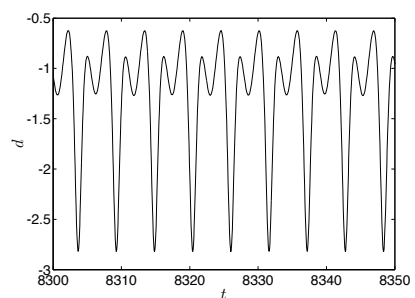

(f)

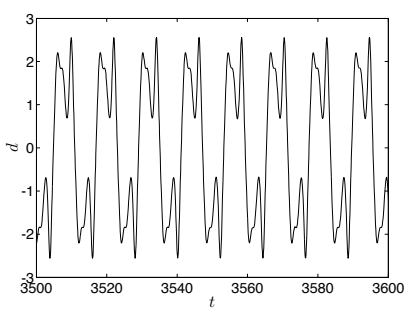

(i)

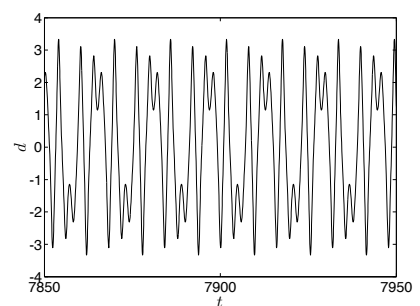

(l)

Figure 13: Time series corresponding to simulations shown in Figure 12: (a) $F=1.515$, $\omega=2.955$, (b) $F=1.995, \omega=0.165$, (c) $F=2.025, \omega=1.155$, (d) $F=2.115, \omega=0.585$, (e) $F=2.895, \omega=2.985$, (f) $F=2.985, \omega=1.125$, (g) $F=1.005, \omega=1.905$, (h) $F=2.085$, $\omega=0.075$, (i) $F=2.655, \omega=2.595$, (j) $F=2.805, \omega=0.795$, (k) $F=2.985, \omega=0.435$, (l) $F=2.985, \omega=1.185$. 


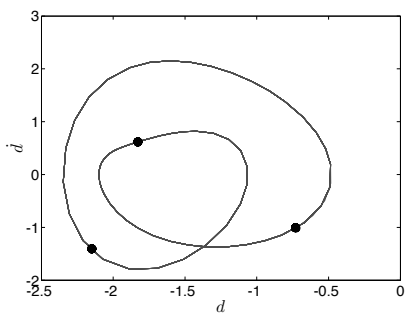

(a)

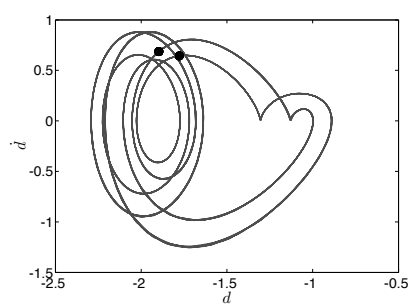

(d)

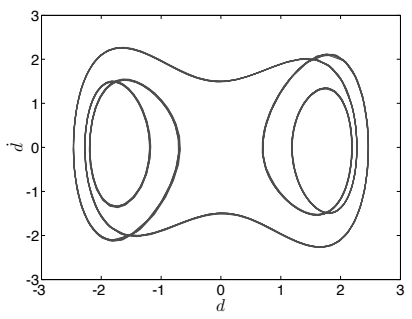

(g)

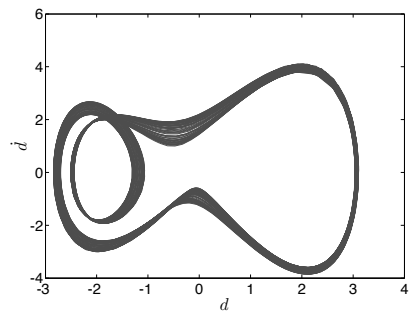

(j)

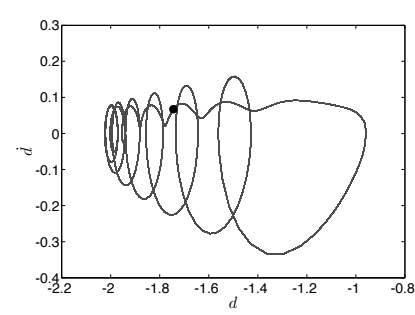

(b)

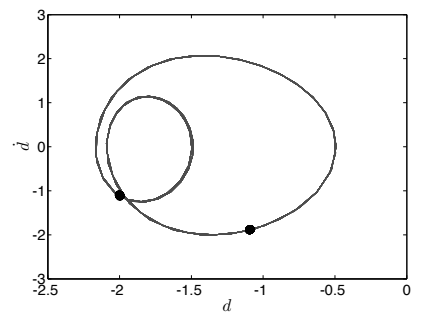

(e)

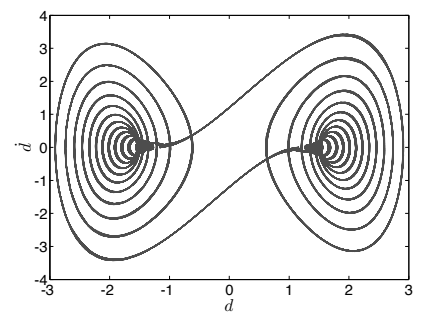

(h)

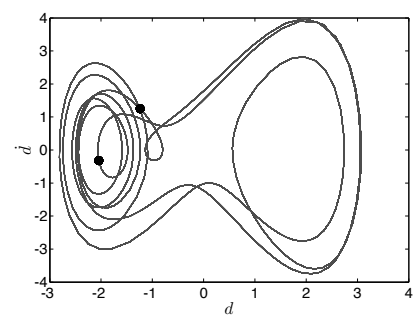

(k)

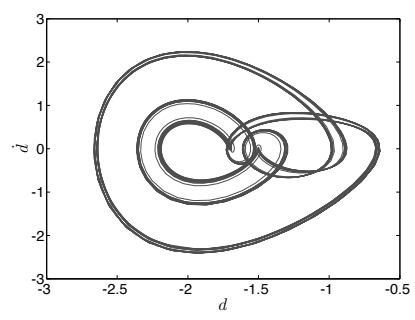

(c)

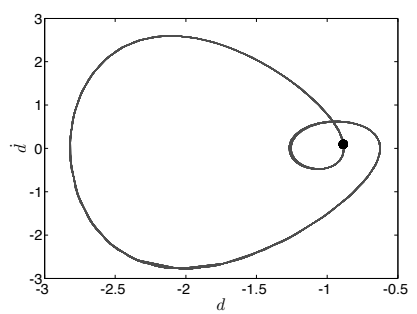

(f)

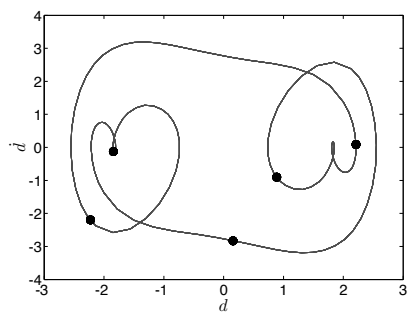

(i)

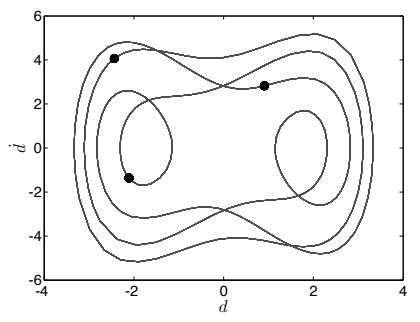

(l)

Figure 14: Phase plots including Poincaré points (black dots) for periodic responses corresponding to cases shown in Figure 12: (a) $F=1.515, \omega=2.955$, (b) $F=1.995, \omega=0.165$, (c) $F=2.025, \omega=1.155$, (d) $F=2.115, \omega=0.585$, (e) $F=2.895, \omega=2.985$, (f) $F=2.985$, $\omega=1.125$, (g) $F=1.005, \omega=1.905$, (h) $F=2.085, \omega=0.075$, (i) $F=2.655, \omega=2.595$, (j) $F=2.805, \omega=0.795$, (k) $F=2.985, \omega=0.435$, (l) $F=2.985, \omega=1.185$. 
forcing period. This produces a discrete mapping that reveals the periodicity (or lack thereof) of the response. If the response is periodic, the Poincaré points are shown in Figure 14 by black dots. Several periodic responses are identified: period one (Figures 14(b) and 14(f)), period two (Figures 14(d), 14(e), and 14(k)), period three (Figures 14(a) and 14(l)), and period five (Figure 14(i)). A system that has a period three response is capable of having a chaotic behavior [14]. Chaotic responses will be discussed later in this section.

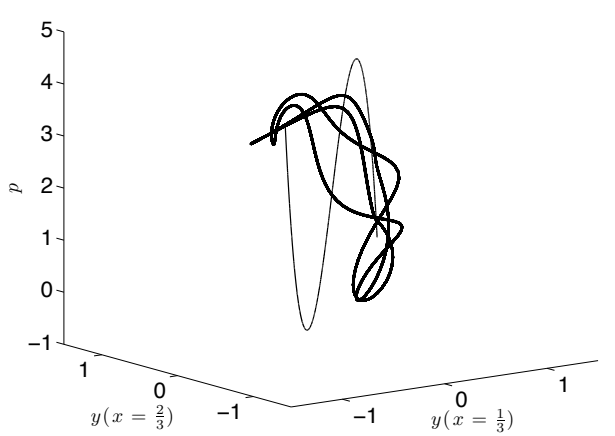

(a)

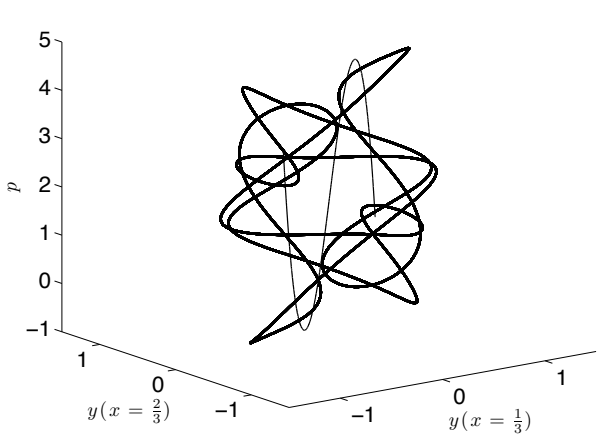

(c)

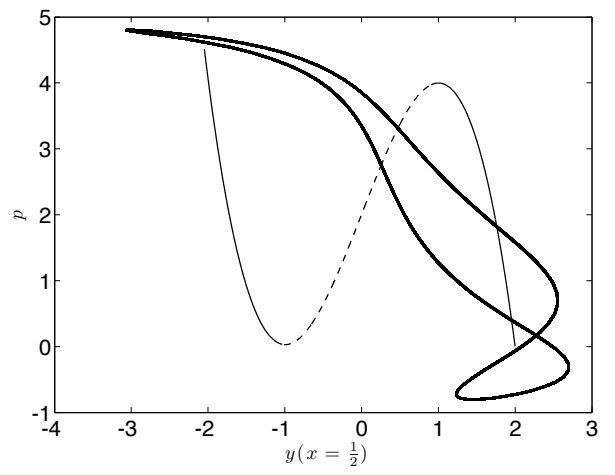

(b)

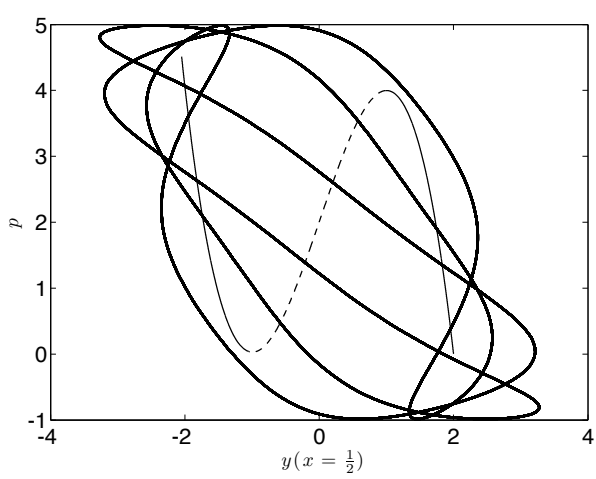

(d)

Figure 15: Asymmetric phase trajectories obtained using the FE model for $\lambda=2$ : (a) asymmetric response for $F=2.805, \omega=0.795$, (b) the projection to the symmetry plane of (a), (c) asymmetric response for $F=2.985, \omega=1.185$, (d) the projection to the symmetry plane of $(c)$. 

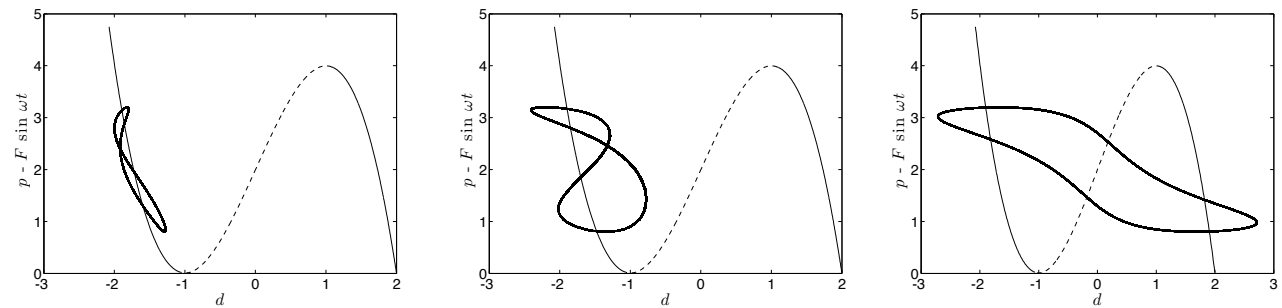

(a)
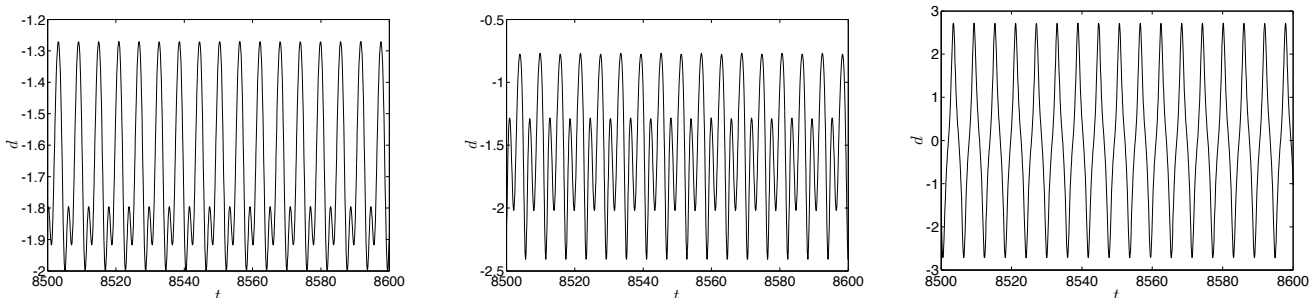

(b)
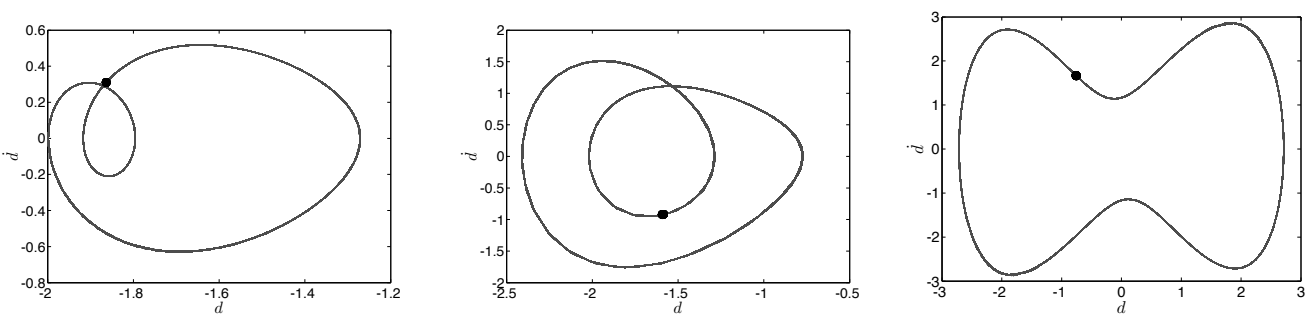

(c)

Figure 16: Snap-through responses for $F=1.2$ and $\omega=1.065$ : (a) phase trajectories of pattern 1, 2, and 3 (from left to right), (b) time series corresponding to (a), and (c) phase plots and Poincaré points corresponding to (a). Two different patterns of inverted snap-through and one persistent snap-through coexist for the same forcing parameters.

Even with the limiting assumption of symmetry in the simplified model, a wide range of post-snap responses is observed in the previous results. Use of a full FE model further enriches this collection: for some values of forcing parameters, non-symmetric responses can be observed, even for $\lambda=2$. Figure 15 shows examples of the phase trajectories of the non-symmetric responses with respect to the displacements at $x=1 / 3$ and $x=2 / 3$ superposed with the equilibrium paths for two pairs of forcing parameters: (1) $F=2.805$, $\omega=0.795$ (Figure 15(a)) and (2) $F=2.985, \omega=1.185$ (Figure 15(c)). These values of the forcing parameters are the same as in Figures $12(\mathrm{j})$ and $12(\mathrm{l})$. 
The asymmetric phase trajectories, obtained from FEM, show that other modes aside from the first symmetric mode are excited and the phase trajectories with respect to displacement at $x=1 / 2$ obtained using the FE model (Figures 15(b) and 15(d)) are different from the trajectories obtained with the one degree of freedom model (Figures 12(j) and 12(l)).
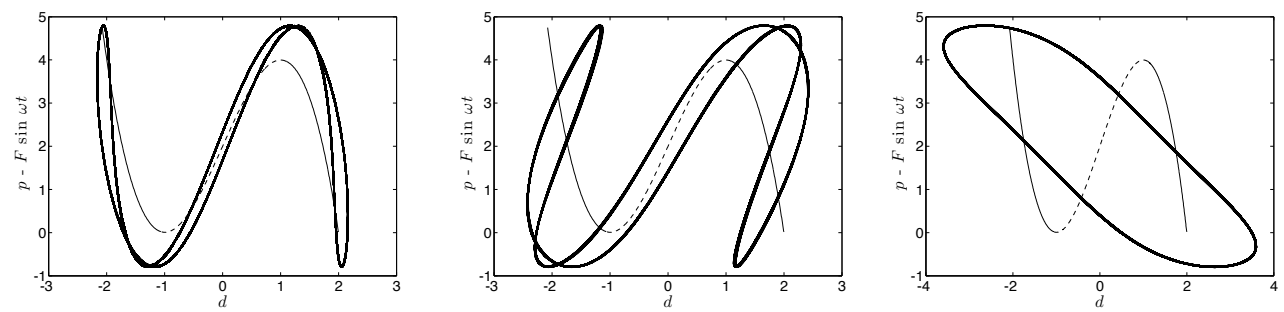

(a)
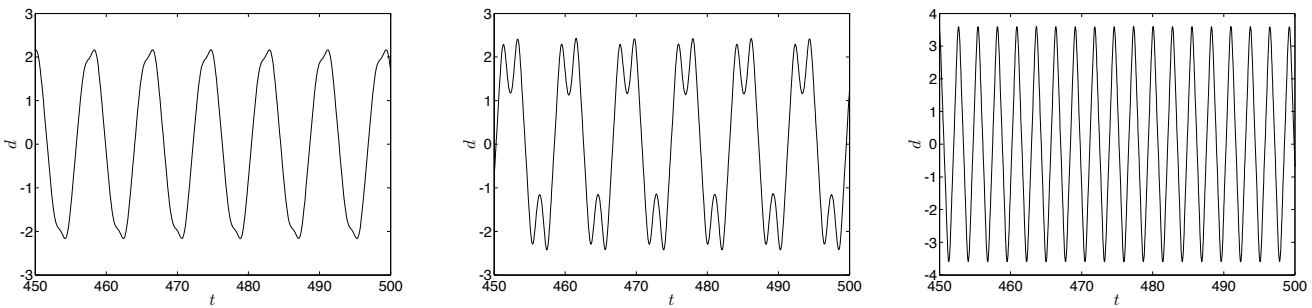

(b)
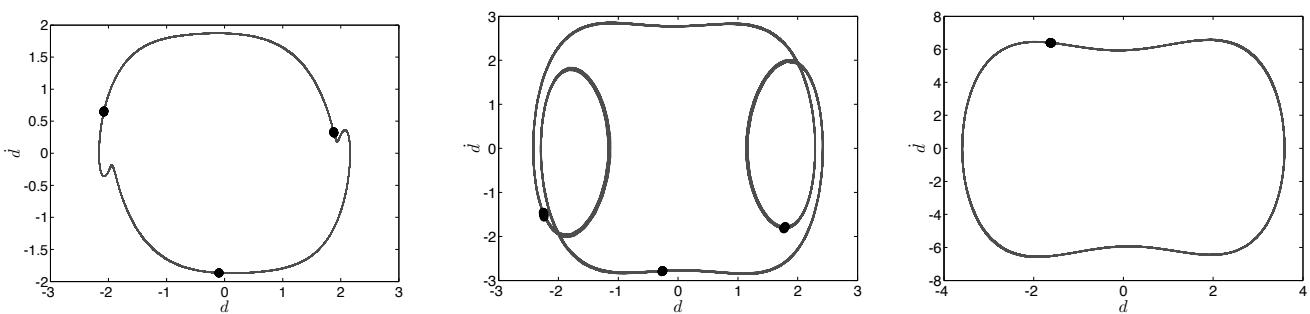

(c)

Figure 17: Different patterns of persistent snap-through responses for $F=2.8, \omega=2.295$ : (a) phase trajectories of pattern 1, 2, and 3 (from left to right), (b) time series corresponding to (a), and (c) phase plots and Poincaré points corresponding to (a). Patterns 1 and 2 show period three responses, pattern 3 shows a period one response.

Previously we showed that, for a given pair of forcing parameters, the arch might undergo different types of snap-through responses under different initial conditions. Further study also shows that the arch might have multiple trajectories of the same snap-through type for a pair $(F, \omega)$ when different 
initial conditions are used, e.g., $F=1.2, \omega=1.065$ (Figure 16) and $F=2.8$, $w=2.295$ (Figure 17). For $F=1.2$ and $\omega=1.065$, five different responses are identified. Three of the responses are shown in Figure 16. The other two responses not shown here are the reverted snap-through versions of the inverted snap-through responses shown in Figure 16(a). Two patterns of inverted snap-through are identified (Figures 16(a)), however they correspond to different amplitude of oscillations.

For $F=2.8$ and $w=2.295$, all initial conditions in the parameter space lead to persistent snap-through scenarios. However, three different patterns of persistent snap-through responses are identified (Figure 17). Even though all responses show persistent snap-through behavior, the time series shown in Figure 17(b) shows that the third pattern has more snap-through events for the same interval and significantly larger amplitudes leading to more fatigue than the other two responses.

\subsection{Snap-through events per forcing cycle}

The number of snap-through events per forcing cycle is a useful quantity for estimating fatigue. Moreover, the plot of the number of snap-through events per forcing cycle versus the frequencies can also be considered a $b i$ furcation diagram. Two different forcing magnitudes are shown: (1) $F=1.2$ (Figure 18(a)) and (2) $F=2.8$ (Figure 18(b)). Each plot contains data for 100 forcing frequencies. Each data point in the plot is obtained from one simulation by averaging the number of snap-through events in 1000 forcing cycles after the transients are allowed to decay. For each frequency, ten simulations with random initial conditions are performed to capture coexisting transient responses for the same frequency. The dashed line in Figure 18(a) identifies the frequency used for the results shown in Figure 16 while the dashed line in Figure 18(b) is at the forcing frequency used in the simulations shown in Figure 17.

When the number of snaps-through per forcing cycle is zero, it corresponds to responses that either do not snap-through at all or do not undergo persistent snap-through in the steady-state region. Cases in which two snapthrough events per forcing cycle occur indicate a period one snap-through where snap-through is in phase with the forcing. When the number of snapsthrough per forcing cycle is neither zero nor two, it corresponds to responses with occasional snaps-through which may occur in higher-period behavior or in chaotic responses. Chaotic responses will be discussed later in this section. 


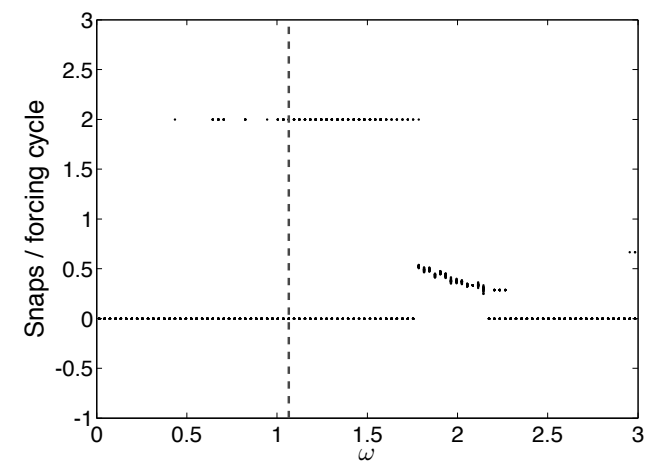

(a)

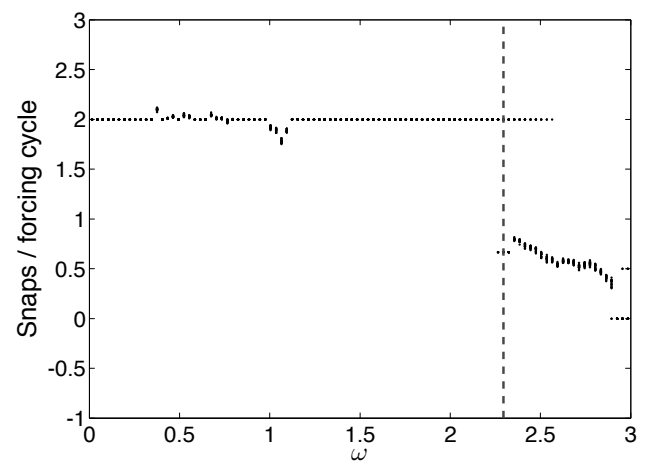

(b)

Figure 18: Number of snap-through events per forcing cycle $\lambda=2$ : (a) $F=1.2$ and (b) $F=2.8$.

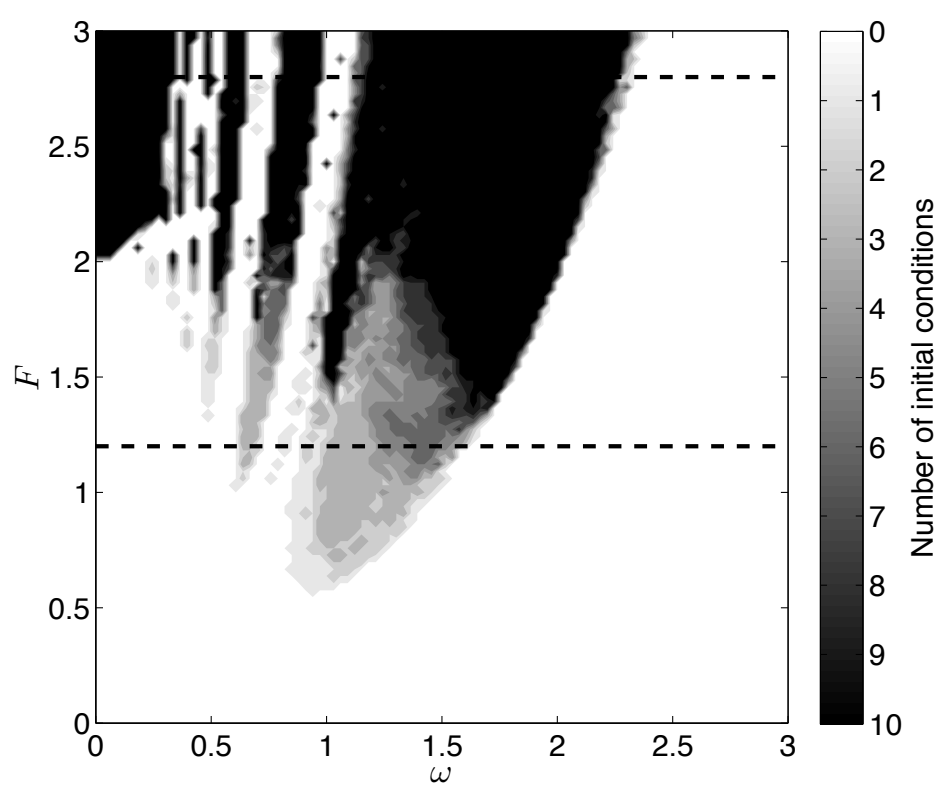

Figure 19: Number of initial conditions leading to period one persistent snap-through responses.

The period one persistent snap-through responses are the most severe conditions for the structure due to the high number of snap-through per forcing cycle. Therefore, identifying the forcing parameters that could lead to this type of response is important. Figure 19 shows the number of initial 
conditions that leads to period one persistent snap-through responses for each pair of forcing parameters for the ten initial conditions used here. The dashed lines in Figure 19 correspond to the forcing amplitudes used in Figure 18.

\subsection{Kinetic Energy}

The kinetic energy can also be used to shed some light on the dynamic behavior of the arch. Figure 20 shows the average kinetic energy for the forcing parameters used in Figure 18. The higher kinetic energy level corresponds to the persistent snap-through case since a system that oscillates between the stable configurations will have higher kinetic energy than a system that oscillates about one of the stable configurations. The kinetic energy can also be used as an additional tool to classify the different responses that occur for the same forcing frequency. The dashed line in Figure 20(a) that corresponds to the frequency used in Figure 16, shows that three different levels of energy are observed for $\omega=1.065$. The responses in Figure 16(a) from left to right correspond to the lowest, mid, and highest levels of kinetic energy, respectively. A similar phenomenon is observed for $F=2.8$ and $\omega=2.295$ (the dashed line in Figure 20(b)): the responses in Figure 17(a) from left to right correspond to the lowest, mid and highest kinetic energy levels, respectively.

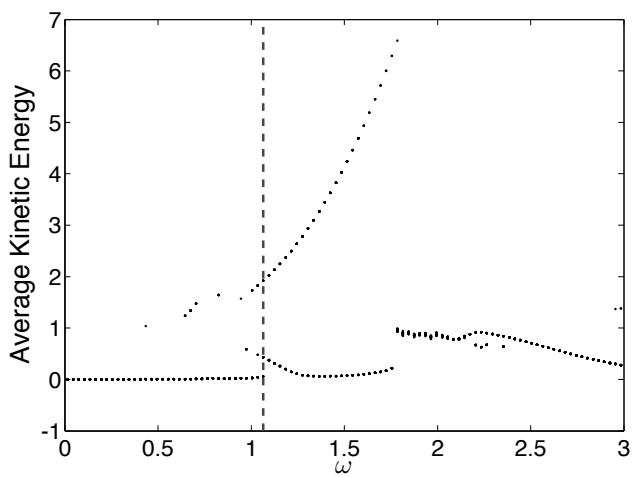

(a)

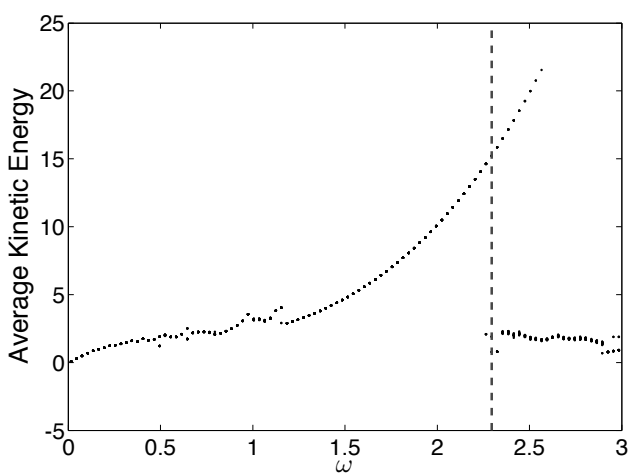

(b)

Figure 20: Average kinetic energy shows the number of different types of responses for each forcing frequency: (a) $F=1.2$ and (b) $F=2.8$.

\subsection{Regions of chaotic responses}

To analyze regions for which chaotic response can occur, the method that is described in [15] is used. This method has been proven to show a 
good agreement with the Lyapunov exponent (LE), a standard method for identifying chaos [2]. The discrete Fourier transform (DFT) of chaotic time series shows a broad spectrum resulting in a higher number of peaks in the DFT in comparison with the DFT for a non chaotic response. By counting the peaks of the DFT above a certain threshold, a measure of chaos can be obtained. The plots of the peak counts for $F=1.2$ (Figure 21(a)) and $F=2.8$ (Figure 21(b)) show clearly that some regions of frequencies have much higher peak counts than others thus identifying the chaotic regions. Examples of chaotic responses are shown in Figure 22. The dashed-dotted lines in Figure 21 correspond to the forcing frequencies used in Figure 22.

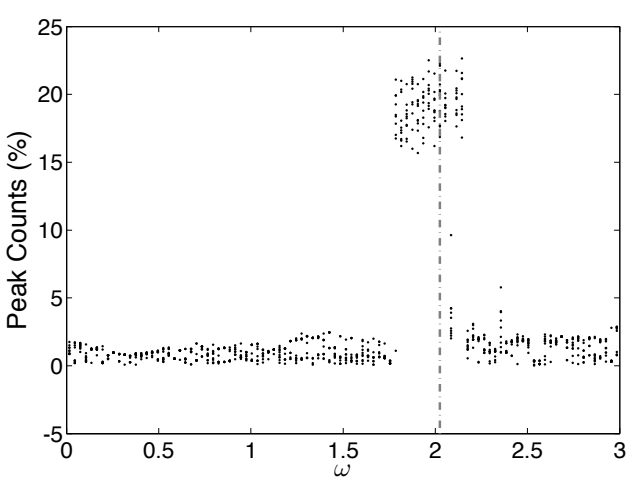

(a)

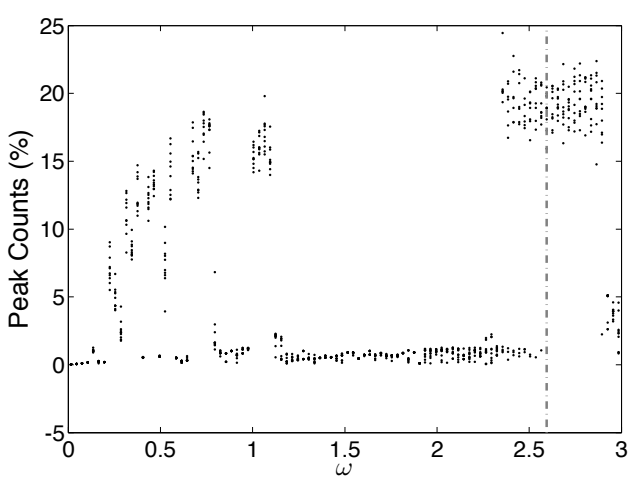

(b)

Figure 21: Percentage of peak count shows the chaotic responses: (a) $F=1.2$ and (b) $F=2.8$.

Figure 23 shows peak counts obtained from the simulations in the $F-\omega$ parameter space. The dashed lines in Figure 23 correspond to the forcing amplitudes used in Figure 21. For each pair of forcing parameters, ten initial conditions are used and the values used in the figure are the maximum peak counts obtained out of all ten simulations. From the figure, we can identify the chaotic region in the $F-\omega$ parameter space.

\section{Conclusions}

This paper presents a numerical investigation of the dynamic behavior of a sinusoidal shallow arch-like system experiencing snap-through under sinusoidal loading. The specific configuration and loading simplify the analysis leading to a single degree of freedom system under some assumptions. The 


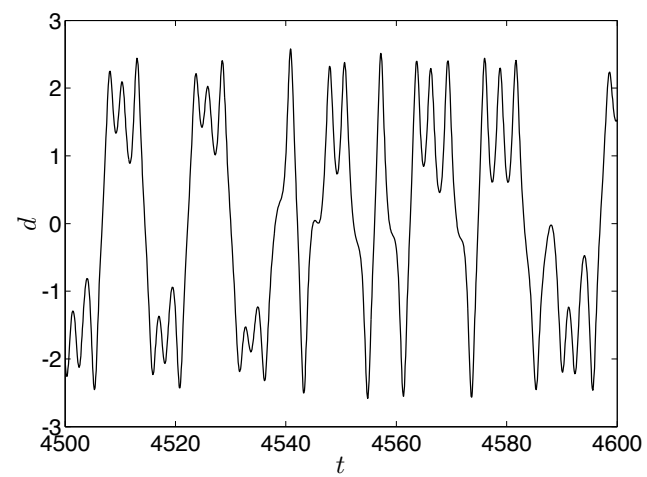

(a)

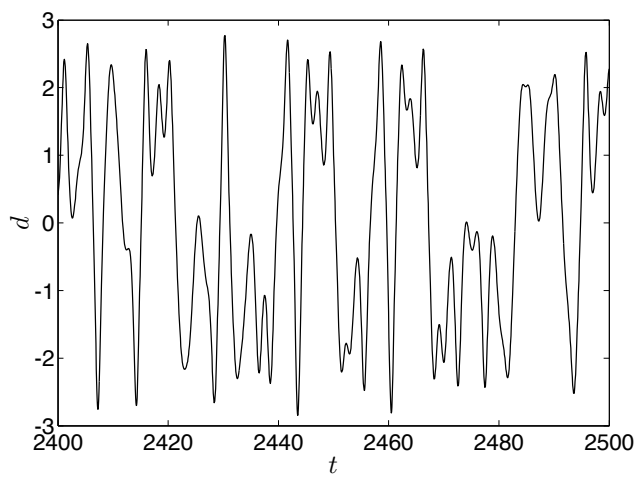

(b)

Figure 22: Examples of chaotic responses: (a) $F=1.2 \omega=2.025$ and (b) $F=2.8$, $\omega=2.595$.

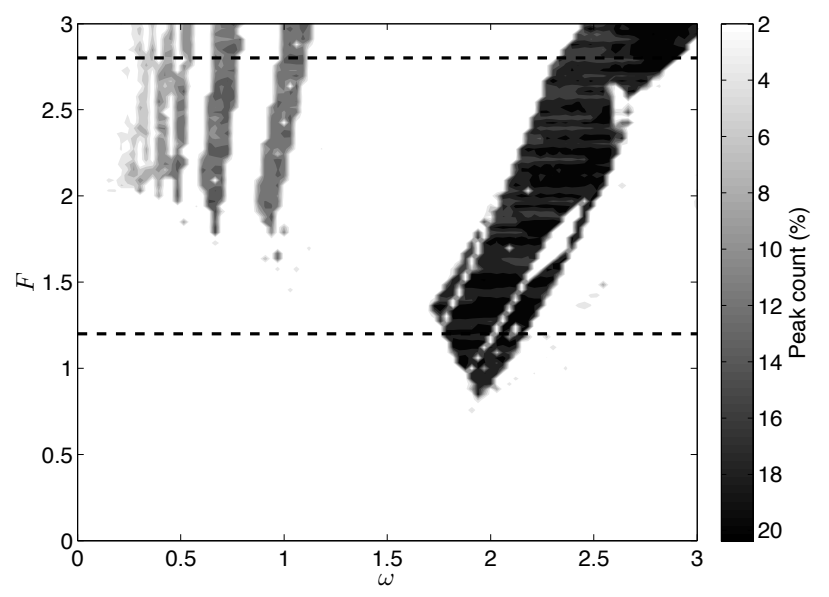

Figure 23: Chaotic region identified using the percentage of peak count in the DFT.

results from the simplified model are then compared with the results obtained from a detailed FE model. We also introduce many useful and illustrative ways to describe relevant features of the snap-through behaviour. This framework for the snap-through analysis can carry over to more general systems. A good understanding of the snap-through behavior is crucial since it increases the risk of fatigue in structures. Typical load-deflection diagrams of shallow arches show that multiple equilibria may coexist and dynamic excitations might cause oscillations between coexisting configurations. The paper focuses on (1) identifying the snap-through boundary of arches under time 
dependent sinusoidal loading and on (2) characterizing and classifying the post-snap responses.

Even though this is a simple structure, the behavior can be quite complex. Depending on the excitation amplitude and frequency, a variety of phase trajectories are observed in the $F-\omega$ space (including chaotic responses). Many are captured with the simplified model but the use of the FE model enriches this collection and shows that for some cases the phase trajectories are asymmetric. Poincaré sections are used as a tool to examine the periodicity of the responses.

Consideration of variability in the initial conditions further increases the complexity of the responses: (1) whether the arch snaps-through, (2) the type of snap-through that occurs, and (3) different responses are obtained for the same type of snap-through. Therefore, the snap-through boundary is likely to expand if more initial conditions are used. The different responses obtained for different initial conditions under the same forcing parameters lead to different effects on the structural fatigue. Therefore, it is important to consider a variety of different initial conditions in order to determine the worst case scenario.

Several tools and measures are used to characterize the transient behavior of shallow sinusoidal arches under different initial conditions: (1) the number of snap-through per forcing cycle, (2) the kinetic energy, and (3) the peak count of the DFT of the response. The number of snap-through and kinetic energy show the different responses that can be obtained for a certain forcing parameters. The peak count of the DFT is used to identify the chaotic regions in the $F-\omega$ space.

\section{Acknowledgements}

The work has been funded in part by AFOSR under the grant no. FA955009-1-0201. This support is greatly appreciated. Support was also received from the Rice University Data Analysis and Visualization Cyberinfrastructure funded by NSF under grant OCI-0959097. 


\section{References}

[1] L. N. Virgin, Vibration of Axially-Loaded Structures, Cambridge University Press, 1st edition, 2007.

[2] R. Wiebe, L. N. Virgin, I. Stanciulescu, S. M. Spottswood, T. G. Eason, Characterizing dynamic transitions associated with snap-through. I: A discrete system, Journal of Computational and Nonlinear Dynamics 8 (2013). DOI:10.1115/1.4006201.

[3] E. Mettler, Stability and vibration problems of mechanical systems under harmonic excitation, in: G. Herrmann (Ed.), Dynamic stability of structures, Pergamon Press, New York, N.Y., 1967, pp. 169-188.

[4] E. Mettler, Dynamic buckling, Handbook of engineering mechanics (1962) 62-61.

[5] C. S. Hsu, Stability of shallow arches against snap-through under timewise step loads, Journal of applied mechanics 35 (1968) 31-39.

[6] J. S. Humphreys, On dynamic snap buckling of shallow arches, AIAA J 4 (1966) 878-886.

[7] M. H. Lock, Snapping of a shallow sinusoidal arch under a step pressure load, AIAA Journal 4 (1966) 1249-1256.

[8] N. C. Huang, Dynamic buckling of some elastic shallow structures subject to periodic loading with high frequency, International Journal of Solids and Structures 8 (1972) 315-326.

[9] W. Y. Poon, C. F. Ng, Y. Y. Lee, Dynamic stability of a curved beam under sinusoidal loading, Proceedings of the Institution of Mechanical Engineers, Part G: Journal of Aerospace Engineering 216 (2002) 209217.

[10] R. H. Plaut, E. R. Johnson, The effects of initial thrust and elastic foundation on the vibration frequencies of a shallow arch, Journal of Sound and Vibration 78 (1981) 565-571.

[11] R. L. Taylor, University of California, Berkeley. Dept. of Civil and Environmental Engineering, FEAP, a Finite Element Analysis Program: Version 8.3 User Manual (2011). 
[12] J.-S. Chen, M.-R. Yang, Vibration and Stability of a Shallow Arch Under a Moving Mass-Dashpot-Spring System, Journal of Vibration and Acoustics 129 (2007) 66.

[13] C. Maurini, J. Pouget, S. Vidoli, Distributed piezoelectric actuation of a bistable buckled beam, European Journal of Mechanics, A/Solids 26 (2007) 837-853.

[14] T. Y. Li, J. A. Yorke, Period three implies chaos, The American Mathematical Monthly 82 (1975) 985-992.

[15] R. Wiebe, L. N. Virgin, A heuristic method for identifying chaos from frequency content, Chaos 22, 013136 (2012). DOI: 10.1063/1.3675624. 


\section{List of Figures}

1 Snap-through buckling of shallow structures: (a) initial and post-snap configuration, (b) load-deflection curve. . . . . . . 2

2 Geometry of a shallow arch with sinusoidal shape and sinusoidal loading. . . . . . . . . . . . . . . . . . . . 4

3 Solution path for $\lambda=2$ : (a) equilibrium path and (b) the first two eigenvalues. . . . . . . . . . . . . . . . . . 7

4 Solution path for $\lambda=2.1$ : (a) equilibrium path and (b) the first two eigenvalues. . . . . . . . . . . . . . 7

5 Solution path for $\lambda=2.3452$ (coincident configuration): (a) equilibrium path and (b) the first two eigenvalues. . . . . . 8

6 Solution path for $\lambda=3$ : (a) equilibrium path and (b) the first two eigenvalues. ................ . . 8

$7 \quad$ Snap-through boundaries and classification of the responses for systems with $\lambda \leq 2.3452$ : (a) $\lambda=2$, (b) $\lambda=2.3452$ (coincident configuration). Similar profiles are observed. . . . . . . 11

8 The number of forcing cycles needed for the arch to snapthrough $(\lambda=2)$. Most cases show the structure snaps-through in less than four forcing cycles. . . . . . . . . . . . . . 12

9 Snap-through boundary determined with the simplified analysis (gray) and full FE model (black). The FE model shows more forcing parameters cause the arch to snap-through in comparison with the one degree of freedom model $(\lambda=3$,

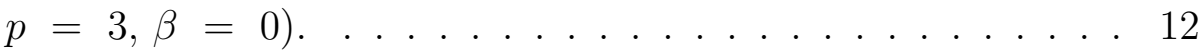

10 Effects of initial conditions on: (a) snap-though boundary, (b) the type of snap-through. Snap-through boundaries expand when more initial conditions are considered. Different initial conditions may result in different types of snap-through for the same forcing parameters. . . . . . . . . . . . . . 14

11 Snap-through boundaries in initial conditions parameters space for $\lambda=2$ : (a) $F=1.2 \omega=0.105$, (b) $F=1.2, \omega=1.515$. . 
12 Time series superimposed on the restoring force $(\lambda=2$; sinusoidal load): (a) $F=1.515, \omega=2.955$, (b) $F=1.995$, $\omega=0.165$, (c) $F=2.025, \omega=1.155$, (d) $F=2.115, \omega=0.585$,

(e) $F=2.895, \omega=2.985$, (f) $F=2.985, \omega=1.125$, (g) $F=1.005$, $\omega=1.905$, (h) $F=2.085, \omega=0.075$, (i) $F=2.655, \omega=2.595$, (j) $F=2.805, \omega=0.795,(\mathrm{k}) F=2.985, \omega=0.435$, (l) $F=2.985$, $\omega=1.185$.

13 Time series corresponding to simulations shown in Figure 12:

(a) $F=1.515, \omega=2.955$, (b) $F=1.995, \omega=0.165$, (c) $F=2.025$, $\omega=1.155$, (d) $F=2.115, \omega=0.585$, (e) $F=2.895, \omega=2.985$,

(f) $F=2.985, \omega=1.125,(\mathrm{~g}) F=1.005, \omega=1.905$, (h) $F=2.085$, $\omega=0.075$, (i) $F=2.655, \omega=2.595$, (j) $F=2.805, \omega=0.795$,

(k) $F=2.985, \omega=0.435,(\mathrm{l}) F=2.985, \omega=1.185$.

14 Phase plots including Poincaré points (black dots) for periodic responses corresponding to cases shown in Figure 12:

(a) $F=1.515, \omega=2.955$, (b) $F=1.995, \omega=0.165$, (c) $F=2.025$, $\omega=1.155$, (d) $F=2.115, \omega=0.585$, (e) $F=2.895, \omega=2.985$,

(f) $F=2.985, \omega=1.125,(\mathrm{~g}) F=1.005, \omega=1.905$, (h) $F=2.085$, $\omega=0.075$, (i) $F=2.655, \omega=2.595$, (j) $F=2.805, \omega=0.795$,

(k) $F=2.985, \omega=0.435$, (l) $F=2.985, \omega=1.185$.

15 Asymmetric phase trajectories obtained using the FE model for $\lambda=2$ : (a) asymmetric response for $F=2.805, \omega=0.795$, (b) the projection to the symmetry plane of (a), (c) asymmetric response for $F=2.985, \omega=1.185$, (d) the projection to the symmetry plane of (c).

16 Snap-through responses for $F=1.2$ and $\omega=1.065$ : (a) phase trajectories of pattern 1, 2, and 3 (from left to right), (b) time series corresponding to (a), and (c) phase plots and Poincaré points corresponding to (a). Two different patterns of inverted snap-through and one persistent snap-through coexist for the same forcing parameters.

17 Different patterns of persistent snap-through responses for $F=$ 2.8, $\omega=2.295$ : (a) phase trajectories of pattern 1,2 , and 3 (from left to right), (b) time series corresponding to (a), and (c) phase plots and Poincaré points corresponding to (a). Patterns 1 and 2 show period three responses, pattern 3 shows a period one response. 
18 Number of snap-through events per forcing cycle $\lambda=2$ : (a) $F=1.2$ and (b) $F=2.8 . \ldots \ldots \ldots \ldots$

19 Number of initial conditions leading to period one persistent snap-through responses. . . . . . . . . . . . . . . . 23

20 Average kinetic energy shows the number of different types of responses for each forcing frequency: (a) $F=1.2$ and (b) $F=2.8 . \ldots \ldots \ldots \ldots \ldots$

21 Percentage of peak count shows the chaotic responses: (a) $F=1.2$ and (b) $F=2.8 \ldots \ldots \ldots \ldots \ldots \ldots \ldots$

22 Examples of chaotic responses: (a) $F=1.2 \omega=2.025$ and (b) $F=2.8, \omega=2.595 \ldots \ldots \ldots \ldots \ldots$

23 Chaotic region identified using the percentage of peak count in the DFT. . . . . . . . . . . . . . . . . . . 26

\section{List of Tables}

1 Dimensions ...................... . . . 6

2 Material properties ............... 6 